which pass at right angles to their surfaces without any foreign material being interposed. A wound thus sutured is closed without any increased number of stitches or stitch-holes and holds the coaptated parts gently at rest.

The method of application of the suture in more common use for the closure of wounds, is the use of the interrupted suture, which necessarily implies a knot for each stitch and can be applied as a deeply buried suture only undor very considerable mechanical disadvantage, not alone involving a difficulty of application, but necessitating a very considerable injury to the structures. Even when used it folds in juxtaposition only the tissues included in the loop of the stitch, and an undue constriction of the included structures is deemed necessary in order to prevent the gaping of the wound between the points of ligation. As a skin suture it has the well recognized disadvantage, already referred to, of penetrating the cuticular layer with its ever-present micrococcus pyogenes albus infection. Here also, to prevent the separation of the wound between the stitches, the suture is made to unduly constrict the enclosed tissues.

The continuous suture in common use (the glover's stitch) has the advantage of rapidity of application and minimization of the suture material, but it is at fault in that the action of the force is not at right angles to the divided surfaces but acts in an oblique direction, which produces a tendency to intrafold and join the opposing surfaces irregularly. By the use of the curved needle the stitches may be introduced from side to side deeply through the uninjured structures of the wound, crossing its cut edges at right angles. When this is gently drawn upon it brings the sides of the wound accurately into apposition without the interposition of suture material. For want of a better term I have called this the parallel suture, since it traverses the structures nearly parallel to the tissues to be coaptated. It is only a slight modification of the so-called blind running stitch in common use by the seamstress, but it fultils a most admirable purpose in surgery.

Although it is remarkable to note how large an amount of aseptic suture may be used with apparent impunity in an aseptic wound, when necessary, it is also surprising to observe how small a quantity of suture muterial is really needful to hold in apposition the surfaces of a large deep wound, as in the amputation of the breast. This stitch is of equal service when applied for the closure of the abdominal wall wi in laparotomy, or in hernia, but for reasons already apparent it must be applied to restore and retain at rest like structures with a minimum of injury to the parts involved. Aseptically applied in aseptic structures and sealed with iodoform collodion the wound will unite, and aseptic primary union without swelling or suffering will supervene.

Agglutinstion as a femostatic Agent.--Lawson Tait is reported to be using at the present writing a new device, which has the credit of much ingenuity. A platinum wire so arranged as to carry a cu.rent of electricity is inclosed in the blades of a pair of steel forceps or any other required instrument, the wire for this purpose being insulated by a bed of burnt pipe-clay. This arrangement being perfected, a current of suitable voltage is turned on, the artery seized and compressed, and in a few sec onds the tissues and arterial walls are so agglutinated that the passage of blood is rendered impossible. The temperature employed is about 180 degrees $F$., the fact being thus apparent that the principle involved in this device is different from that of electric cauterizing instruments.
TWO HUNDRED AND FIFTY BASSINI OPER. ATIONS FOR THE CURE OF INGUINAL HERNIA; WITHOUT MORTALITY.

Presented to the Section on Surgery and Anatomy, at the Forty-eighth Annual Meting of the American Medical Association, at

Philadelphia, Pa., June 1-4, 1897.

BY W. B. DE GARMO, M.D.

PROFESSOR OF SPECIAL SURGERY, NEW YORK POST-GRADUATE MEDICAL SCHOOL AND HOSPITAL.

$$
\text { NEW YORK. }
$$

The 250 operations here reported have been done upon 216 patients; 34 of the cases having been operated on on both sides. Fifty-two of the patients were females, 164 were males. I am quite sure that no one can accuse me of having selected my cases, when their ages and conditions are considered. Fiftyfive of the patients were under 14 years of age. 43 were between 14 and 25 , while 118 had passed their 25 th year. Of the latter number, 8 were between 60 and 70,6 between 70 and 80 and 2 were over 80 years of age. The youngest child operated on was $\tilde{5}$ months old, and this is the first instance in my experience where I have been obliged to operate on a child less than 1 year of age. The operation was for strangulated scrotal hernia on the right side. The left was operated on at the same time for a large scrotal hernia, and the child made a perfect recovery in ten days.

The age limit for operation on these cases does not seem to have been reached in either direction, as the oldest patients operated on seemed to stand the operation as well, and recover as promptly as those operated on in early life.

Ninety-three of the cases had scrotal hernia; 55 were irreducible, 17 were strangulated and 178 were reducible at the time of the operation. In fifty-five of the cases it was necessary to excise more or less omen. tum, and this was done by tying off with multiple silk ligatures, in the manner described by me in the Annals of Surgery, June 2, 1895. I am not disposed to change this method now that I have a total of seventy cases without a death, which seems to prove its efficiency and safety.

The largest hernia operated on was two feet in circumference, reaching two-thirds of the distance to the knee of the patient, who was a man 53 years old. The tumor contained large and small intestine, a quantity of omentum and a distended bladder. This man had told me that he had difficulty in urinating unless he pressed the tumor forcibly against his thigh with his hands, but the exact condition of the bladder did not occur to me until after the operation. Twentyfour hours later it was found that he was passing very little urine and as the catheter could not be introduced, the bladder was becoming enormously distended. Forty-eight hours after the operation the bladder was therefore opened through the perineum and free drainage established. Notwithstanding the extent of the secondary work done upon him, the man recovered from his hernia operation by primary union and has now remained cured for two years.

The success of the Bassini operation is believed to be largely due to two facts: That all abnormal struc. tures ure cleared out of the canal; that two muscular layers are brought down to form a new posterior wall. Aside from the hernial sac and its contents, I have found in the canal in three instances the ovary; in nine cases the testicles, and enlarged veins in a number of males. A young woman 30 years of age, accus. tomed to all athletic sports and in perfect health, had 
in both canals masses of varicose veins having every appearance of extreme varicocele as found in the male. These were ligated and cut away, but their cause and actual source of supply remained unknown.

Bunches of extra-peritoneal fut have been frequently met with in the canal in masses larger than one's thumb, and in one case as large as a good sized hen's egg. This man had scrotal hernia on one side and was supposed to have complete inguinal hernia on the other, but no hernial sac was found, merely this mass of fat coming down in front of the peritoneum. It was loose in the canal, hanging by a pedicle. and he could therefore push it up above a truss pad that he had been accustomed to wear.

It is not a very uncommon thing to have men tell me that formerly they were fat, and upon getting thin they have developed hernia. Doubtless, these are cases of the character just described, where previously the canal had been dilated by extra-peritoneal fut, and when this was absorbed a true hernia had come through the canal. I am quite sure that cases of this character are a far more common source of hernia than is generally supposed.

Cysts have been found in the canal in four cases and this does not apply to those frequently found in the scrotum just above the testicle. In one case it took the form of multiple small cysts, the whole resembling a small bunch of white grapes; this in addition to $\mathrm{a}$ well developed scrotal hernia. The length of time that these cuses have been under treatment is of interest and importance. The time required for the complete healing of the case and the day upon which the patient first leaves his room or the hospital is considered the day of discharge.

Of the 250 cases 207 healed by primary union, and these patients were allowed to leave their beds by the tenth day, and in most instances left the hospital by the fourteenth day. Fifty-three healed by secondary union. The shortest stay in the hospital was eleven days. This was following an operation on a physician who, on his own responsibility during my absence from town. left the hospital and took a train for Detroit on the evening of the eleventh day. I have been assured by him that he suffered no inconvenience, nor did it seem to compromise the result in the least. I have only had six patients out of the total number who have required more than twentyeight days for complete recovery.

In the suppurative cases the infection has seemed in almost every instance to start immediately beneath the skin and has not extended to the deeper tissues. In one instance the wound became infected by a gonorrheal discharge, the existence of which was not known at the time of the operation.

Fat patients, whose skins have been abraded by truss wearing, are peculiarly liable to suppuration, even when great care has been taken in the preparation for operation.

Contrary to my own expectation, in but a single instance has recurrence taken place among those cases in which there was suppuration, and in this one it is known that the failure was in no way due to a lack of primary union.

Suppuration in children under 14 years of age has been seen in only one instance in fifty-five cases; children, apparently, not being at all liable to this accident. In the case noted it was a second operation where there was considerable matting together of the tissues.
The donble operations were in every instance done at the same time, as also some cases of inguinal and umbilical hernia. One patient, a man of 52 years, was operated on for double inguinal and right femoral hernia on the same day and has remained cured of the three hernias nearly two years.

Suture matericul. - In my early operations by this method I did not have faith in the lasting properties of kangaroo tendon or chromicized catgut, and from my experience with silk I was somewhat suspicious of that and was therefore led to use silkworm gut for closing the aponeurosis of the external oblique. This was used in twelve cases and in four of these considerable trouble was afterward experienced by the sutures coming out. In eight of the cases they were perfectly retained and have caused no irritation of the tissues. In one instance sutures came out nearly one year after the operation. This material was, therefore, entirely abandoned and since then I have used kangaroo tendon exclusively. In none of these cases, however, has there been a recurrence of hernia, even when the sutures came out. One man was operated on two years since where ordinary catgut was the suture material. No recurrence has taken place.

I am not prepared to disprove the statement of some of my friends that catgut, chromicized or plain, is just as good as kangaroo tendon, but prefer that they should first show an equal number of cures before I change.

One thing in connection with the presentation of this series of cases, I feel that I have reason to congratulate myself upon, and that is that there have been no deaths. I have not gone to the trouble to look over the literature on the subject, but I believe this to be the largest number of operations ever reported for the cure of hernia without a single death.

In one respect I may be liable to criticism on this point, for while I report seventeen cases operated on when the hernia was strangulated and when recovery and cures were effected $I$ do not report two fatal cases operated on during the same period of time. This report relates to the Bassini operation for the cure of inguinal hernia, and has nothing to do with the fatality of strangulated hernia. If the canal has been closed by the Bassini method and the patient survives, then certainly it belongs to this report; otherwise, not.

Next in importance to its safety is the consideration of the permanence of the cure. In a paper on this subject, before this body, at Baltimore, in 1895, I ventured to predict that the permanent cures would be 90 per cent. I am convinced that this estimate is too low, and do not hesitate to advance it to 95 per cent., with five years duration of cures after the operation, but this statement is premature and $I$ am not here to argue this point, but to state the facts as they exist and allow each one of my hearers to draw his own conclusions. None of the present series of cases have been operated on more than three years. One hundred and fifty of them, however, have gone over one year since the operation and in no case, except of actual reccurrence, has a truss been applied. Out of the 250 operations I have had six recurrences. Three others are under supicion, but not sufficiently so to warrant the application of a truss. Of the six recurrences three have been reoperated on, and apparently with success. This leaves only three actual recur-

1 "A Note on the Advance in the Surgical Cure of Hernia," Jourmal of the American Medical Association, July 13,1895. 
rences. By notes made at the time of the first operations it is believed that the real cause of failure has been known in every case. For a clear comprehension of the subject these cases must be briefly considered.

Case 1.-My first recurrence was in a perfectly healthy young man 20 years of age, operated upon on June 3, 1895, for scrotal hernia of recent origin. The sac was tied off at the peritoneal surface and removed and the operation completed in the usual manner. He left the hospital on the fourteenth day, perfectly healed and four months later fell into an open trap doorway, falling violently upon his abdomen across the edge of the floor. One week later a bulging across the line of incision, and he was reoperated on Feb. 6, 1897, so far with apparent success.

Case 2.-The second recurrence was in a boy 6 years old, operated on for congenital hernia June 4, 1895. Primary union months later he developed a small protrusion at the line of the incision while struggling violently with the family physician and the father, who were trying to remove a piece of wood from the urethra. Reoperated upon Feb. 15, 1897, with apparent success.

Case 3.-The third recurrence was in a man 39 years of age, who had a large and troublesome hernia for many years. Operation Nov. 8, 1895; he left the hospital on the fourteenth day, completely healed. This patient was not seen until after his entrance for the operation, and it was not discovered that he suffered from an enormous varicocele on the same side. It was noticed at the time of the operation that the cord was large, but nothing was done for it. As soon as the man was on his feet it was seen that the varicocele was very liable to reproduce the hernia, which it did within six months of his leaving the hos. pital. Reoperated on May 11, 1897. The varicocele and all abnormal veins in the canal were removed. The cord was found to be fully as large as one's finger and was reduced to about the size of a lead pencil. This man has just left the hospital entirely healed. There is no apparent change in the circula tion in the testicle after this charge in the size of the cord.

Case 4.-Man, 37 years of age, weighing 225 pounds, had left irreducible scrotal hernia the size of a small cocoanut. Upon first examination believed to be a mass of omentum; the operation revealed the fact that it was largely bowel. Proved to be the sigmoid flexure turned out through the canal without peritoneal covering. Had not the opening been made to the internal ring it would have been mistaken for the hernial sac and opened. This man had fatty degeneration of the abdominal muscles to such an extent that the sutures would tear through the tissues readily under moderate tension. Hernia gave evidence of recurring in about six months and a light truss was applied and will be continued. Second operation not advised.

Case 5.-Man, 40 years old, six feet five inches tall, weighing 225 pounds, but not healthy looking, had had left scrotal hernia since early childhood, and irreducible for many years. A large mass of hypertrophied omentum was removed. Case was very difficult for operation and in closing the structures, by mistake, the aponeurosis of the external was carried under the cord for a part of the distance on the first row of sutures. On account of the tedious previous work and the condition of the patient time was not taken to correct this. Recurrence in three months.

Case 6.- Man, 55 years of age, of frail build but apparently good health, had had right inguinal hernia for several years, which proved rather rebellious under truss treatment. $\mathrm{He}$ took ether nicely, but after the canal was opened seemed to collapse, pulse dropping to 40 and respiration very feeble. Omentum firmly adherent to upper part of the canal. The necessity for a prompt termination of the operation seemed evident, and the upper part of the sac with adherent omentum was inverted; a suture of kangaroo tendon was placed so as to bring the peritoneum, transversalis and internal oblique as closely around the cord as possible, and the suturing of Poupart's ligament to the deep muscular structures was done in the usual way, except that it was hurriedly done. The patient was only on the table twenty-five minutes and came promptly out of the ether in good condition; a small quantity of ether had been judiciously administered. Patient made a rapid recovery, but showed evidence of recurrence within three months. Light truss applied and now being worn.

Thus, of my six recurrences, two were caused by unusual violence, two on account of undue haste in closing the canal because of the condition of the patient. One was a sigmoid hernia where there was a fatty degeneration of all muscular structures. One was undoubtedly reproduced by a very large varicocele overlooked at the time of the operation.

Three of these cases have already been operated on and, it is believed, cured. Of the remaining three, it is believed that only one is absolutely incurable, and that is the one with fatty degeneration. They are all vastly improved over their condition previous to the operation.

In closing, I feel that I can safely say, that by the Bassini operation and the present advanced methods of surgery, inguinal hernia has been taken from the list of incurable and placed on the list of curable affections.

Operations, 250 ; patients, 216 ; both sides, 34 ; females, 52 ; males, 164.

Under 14 years of age, $55 ; 14$ to 25 years of age, $43 ; 25$ to 40 years of age, $55 ; 40$ to 50 years of age, $29 ; 50$ to 60 years of age, $18 ; 60$ to 70 years of age, $8 ; 70$ to 80 years of age, 6 ; over 80,2 . Position.--Right, 154 ; left, 96 ; Double, 34 .

Kinds.-Scrotal, 93 ; reducible, 178 ; irreducible, 55 ; strangulated, 17 ; retained testicle, 9 ; displaced ovary, 3.

Method of healing.-Primary union, 207 ; secondary union, 43. Omentum was excised in 55.

Cures, 244 (recurrences, 6) ; reoperated, 3. Total, 247.

Discharged.-Shortest time 11 days, longest time 84 days. By the fourteenth day, 114 ; twenty-first day, 84 ; twenty eighth day, 12 ; thirty-fifth day, 4 ; fifty-sixth day, 1 ; eightyfourth day, 1 .

56 West Thirty sixth St., New York. Discussion.

Dr. H. O. MARCy of Boston-I wish to point out the essen tials as to the cause of cure from an anatomic standpoint. The reason that we are not all the subjects of hernia is because the inguinal canal is at a right angle to the intra-abdominal pressure. I have operated more than four hundred times, sometimes as early as three months, and as late as 80 years. During all this experience, even where the intestine itself has been involved, I have never seen a case approach the danger line. I am sure that over 90 per cent. of my cases have remained permanently cured, and I consider all other operations are hardly worthy of discussion. You should all become familiar with the technique of this operation and with the details referable to it in practice. There are three or four million trusses worn in this country alone, and these people should be restored to usefulness. It can be done and should be done. As to the age, I have operated on over thirty patients beyond 60 years of age, and they have improved and been cured as easily as anyone else. Those in middle life and young people should always be operated on.

Dr. A. J. OchsNer of Chicago-I wish to mention the age limit. It is apparent to everyone who sees many hernias that patients will do very well with a truss until they become afflicted with urinary disturbance, and then they will begin to suffer and their truss will no longer be satisfactory. When Dr. White's paper on the "Treatment of Castration" was published it occurred to me that it would be a wise thing in these cases, instead of doing the Bassini operation, to make a section of the cord or to castrate on the side of the hernia. In these cases I think that castration or a ligature of the cord on the side of the hernia would have the same result as Dr. White has noticed in his cases. He has found that urination has been made easy while the difficulties from which the patient had suffered previously all disappeared. In addition, the increased pressure on the hernial ring was also decreased. It is too early yet to consider these cases as permanent cures. For many years I have treated many hundred little boys with inguinal hernixe and have found that many of the cases also suffer from phimosis. In every case less than twelve years of age it can be cured without herniotomy, but by simple circumcision and placing the patient in bed for several weeks afterward with the foot of the bed elevated at an angle of 45 degrees. I do not think we should operate on children under 12 years of age unless this condition has been considered, and I think the time limit important.

Dr. B. MerRiLe Ricketts of Cincinnati-I would like to ask if the members have ever come in contact with hydrocele and undescended teaticle.

Dr. W. Frank Mayo of Rochester, Minn.--There are some things about the Bassini operation which are somewhat differ- 
ent from that of Dr. Marcy. It has always seemed to me that the important point in the Bassini operation was the method of placing the sutures. His method of suturing the canal in a single layer from the inside to the outside is liable to failure without some such suture as the metallic suture. Bassini's operation divides the cord. I would like to call attention to the catgut which has been introduced in these cases. The cases which have relapsed have always been cases which have suppurated. Dr. DeGarmo states that he has had only one case suppurate in children. This has been my own experience and I suppose it is because the parts are more resistant, although there are a certain number in which suppuration will certainly take place.

Dr. E. D. Ferguson of Troy--If the suppuration is super. ficial and does not involve the fibrous layers of the skin, your results will be good; if it is deep and does involve the fibrous layers it will probably fail. If you use the metallic suture it is permanent. We want sutures to last a certain length of time, and they should hold the parts together thoroughly for several weeks, but at the same time they should not be a permanent suture. Whatever the nature of the suture may be, it is a question of restoring the obliquity of the canal for a sufficient length of time to establish firmness, and a question as to whether a certain suture may be used is a matter of minor importance. The question of establishing the union of the fibrous layer on the posterior wall of the canal, especially when pressure comes from the abdominal side, is an important one. This is what Dr. Marcy claimed before Bassini operated at all.

Dr. DeGarmo of New York City - We are indebted to Dr Marcy for his demonstration as to the causes of hernia. There is a question about his having preceded Bassini in the opera tion of restoring the obliquity of the inguinal canal, but so far as I know Dr. Marcy was the first one to do this. Bassini use silk in his operation. I have twice excised the testicle on the side of the hernia. As to phimosis, twenty years ago there was something written on this point. If a child has phimosis circumcision should be done. If we go to a Hebrew institution where the children have been circumcised in early life we will find as many cases of hernia as anywhere else, but I do not wish today to advocate operating on infants in all cases, although it will be well to do it in many. Many children 7 or 8 years of age can not be cured by a truss and they should be operated on. I have seen hydrocele associated with unde scended testicle and it was as large as a hen's egg. In this case the testicle was brought down, but I have seen instances of undescended testicle where the cord was too short. I merely clear the space outside of the perineum and cover muscular tissue over it. In one man there was very marked relief by this method, but if the cord is long enough the testicle should always be brought down. We are perfectly safe in leaving the testicle outside of the peritoneal cavity and in the muscular structure. Tendinous structures need about six weeks for union, and I would like to use something which is not absorbed, but this is difficult to do. Kangaroo tendon suit me very well.

\section{ON THE TECHNIQUE OF PNEUMOTOMY}

Presented to the Section on Surgery and Anatomy at the Forty-eighth Annual Meeting of the American Medical Association, held at Philadelphia, Pa., June 1-4. 1897. BY CARL BECK, M.D.

1'ROYESSOR OF SURGERY, NEW YORK SCHOOL OF CLINICAL MEDICINE; SUREON TO ST. MARK'S HOSPITAL, ETC. NEW YORK, N. Y.

While the surgical principle, "Ubi pus, ibi evacuc,", is nowadays held up more than ever before even in those parts of the human body that are accessible only under great difficulties, there seems to be some exception in regard to pus accumulations in the lungs, although they are by no means of rare occurrence. This lack of enthusiasm in attacking lung abscesses by the surgical knife is apparently caused by the widespread prejudice that they are all of a tubercular character and could consequently not be cured by simple evacuation. But while there is no doubt that the presence of one tubercular abscess necessarily presupposes the affection of a more or less extensive area of lung tissue, the nature of which would certainly be but little influenced by the opening of the single abscess, still there are many abscesses which are caused by preceding inflammatory processes, by suppurative bronchitis, bronchiectasy, etc. These are of a non-tubercular character and are curable, if treated after true surgical principles. If this fact were fully realized, the medicamentous armamentarium of euthanasia would be given up in many cases of alleged phthisis. And here is the salient point in the difficulty of the diagnosis. Still, tine diagnosis of the presence of lung abscess is much easier than its localization.

The presence of copious purulent expectoration, its admixture of elastic fibers and blood pigment, the history of a preceding inflammatory process, particularly of pneumonia, which ran no typical course, the physical signs of the presence of a cavity, the absence of tubercular manifestations, etc., should point to the existence of a lung abscess. As to localization, it has to be borne in mind that while cavities of the apex contain more or less air, those situated further below contain purulent secretion only. If in the latter variety expectoration is copious, so that the cavity becomes evacuated, the respiratory sounds become tympanitic on percussion and are well perceptible on auscultation. If, on the contrary, the cavity is filled up, there is complete dulness and the respiratory sounds are hardly, if at all, audible, pectoral fremitus also being entirely absent.

Cavities of recent origin are easier localized than old cases, not only because the course of the precursory disease furnishes some elucidation, but also because the physical symptoms are much more clearly pronounced. Old cavities are, with few exceptions, deeply situated and can, according to clinical experience, generally be reached below the lower angle of the scapula.

Exploratory puncture, while absolutely reliable in pyothorax, often fails to disclose lung abscess and has, therefore, to be replaced by exploratory pleuro- or pneumotomy. (Compare the writer's article on exploratory pleurotomy and resection of costal pleura, $N$. $Y$. Medical Journal, June 15, 1895.)

The principles of treatment are governed by the same as those which are determining in any case of abscess, that is, thorough evacuation and drainage. This can only be done well by making a wide opening in the chest wall. To accomplish this the resection of at least two, preferably of three or four ribs is required.

The technique is as follows: Thorough asepsis is just as necessary as in any other operation. Particular attention must be given to the skin of the patient and to the hands of the surgeon, scrubbing with green soap first for three or four minutes, then washing with alcohol or ether, and subsequently with bichlorid, 1 to 500. To sterilize the skin of the patient thoroughly, it is advisable to cover the field of the operation with a poultice of green soap. If there is enough time, the poultice may remain for twenty-four hours. I regard this an essential factor for the disinfection of the skin, because I do not believe that under ordinary circumstances the epidermis, which shelters a multitude of pathogenic bacteria, can be rendered sterile by the usual methods of disinfection, which are generally not carried out longer than from ten to fifteen minutes. A period of twenty-four hours gives the soap a chance to permeate the epidermis thoroughly, so that scrubbing on the following day is much more effective. Sometimes indeed the poultice macerates the epidermis so that it can be 